\title{
Os profissionais de saúde e o exercício dos direitos humanos por portadores de transtornos mentais
}

\author{
Healthcare professionals and the practice of the human rights of individuals with mental disorders
}

Profesionales de salud y el ejercicio de los derechos humanos de pacientes con transtornos mentales

Carla Aparecida Arena Ventura ${ }^{1}$, Viviana Carolina Oyan de Moraes², Márjore Serena Jorge ${ }^{3}$

\footnotetext{
${ }^{1}$ Bacharel em Direito, Doutora em Administração. Professora Associada da Escola de Enfermagem de Ribeirão Preto (EERP) da Universidade de São Paulo (USP). Ribeirão Preto, São Paulo, Brasil. E-mail: caaventu@gmail.com.

${ }^{2}$ Acadêmica do curso de graduação em Enfermagem da EERP/USP. Ribeirão Preto, São Paulo, Brasil. E-mail: vivianabtu@hotmail.com.

${ }^{3}$ Enfermeira. Discente do Programa de Pós-Graduação em Enfermagem Psiquiátrica, nível Mestrado, da EERP/USP. Ribeirão Preto, São Paulo, Brasil. Email: marjore.serena@hotmail.com.
}

\section{RESUMO}

Estudo qualitativo cujo objetivo foi descrever a percepção de profissionais de saúde de um Centro de Atenção Psicossocial (CAPS) sobre os direitos humanos dos portadores de transtornos mentais e os meios para efetivação desses direitos durante o cuidado à saúde. Os dados foram coletados em 2010 por meio de entrevistas semi-estruturadas com sete profissionais de saúde e analisados por meio de análise de conteúdo. Foram observadas diferentes percepções sobre os direitos humanos dos portadores de transtornos mentais com enfoques centrados na liberdade, proteção à vida, convivência em sociedade e exercício da cidadania. Apesar dos avanços políticos, foram identificadas lacunas para a consolidação dos direitos dos portadores de transtornos mentais, que podem ser supridas, especialmente, por meio do envolvimento da família, sociedade e governo. Os profissionais de saúde tem assumido importante papel como disseminadores de informações sobre direitos, participando da conscientização dos usuários dos serviços e suas famílias e buscando a reinserção social.

Descritores: Direitos Humanos; Direito à Saúde; Pessoas Mentalmente Doentes; Saúde Mental.

\section{ABSTRACT}

This qualitative study aimed at describing the perception that healthcare professionals of a Psychosocial Care Center (CAPS, as per its acronym in Portuguese) make of the human rights of individuals with mental health disorders and the means used to make rights effective in health care. Data were collected in 2010 by semi-structured interviews with seven professionals, and submitted to content analysis. It was observed that subjects have different perceptions regarding the human rights of individuals with mental health disorders, centered on freedom, protection of life, living in society and being a participative citizen. Despite the political advancements, gaps still hinder making the rights of people with mental disorders effective, which could be supplied by an engagement of the family, society, and government. Healthcare professionals have played an important role as of regarding rights, helping to raise awareness of patients and their families, aiming at their social reinsertion. Descriptors: Human Rights; Right to Health; Mentally Ill Persons; Mental Health.

\section{RESUMEN}

Estudio cualitativo objetivando describir la percepción de profesionales de salud de Centro de Atención Psicosocial (CAPS) sobre derechos humanos de pacientes con transtornos mentales, y los medios para efectivizar tales derechos durante el cuidado de salud. Datos recolectados durante 2010 mediante entrevistas semiestructuradas con siete profesionales de salud, analizados según análisis de contenido. Fueron observadas diferentes percepciones sobre derechos humanos de pacientes con transtornos mentales., con enfoques centrados en la libertad, protección de la vida, convivencia en sociedad y ejercicio de la ciudadanía. A pesar de los avance políticos, fueron identificados vacíos en la consolidación de los derechos de pacientes con transtornos mentales, que podrían salvarse, particularmente, con la participación familiar, de la sociedad y del gobierno. Los profesionales de salud han asumido un importante rol como divulgadores de información sobre derechos, participando en la concientización de los pacientes y sus familias, buscando la reinserción social.

Descriptores: Derechos Humanos; Derecho a la Salud; Enfermos Mentales; Salud Mental. 


\section{INTRODUÇÃO}

Os direitos humanos representam um processo de construção social embasados no ideal de participação de toda pessoa na vida em sociedade ${ }^{(1)}$. Contudo, alguns grupos da população possuem mais dificuldades para exercitar os seus direitos. Os portadores de transtornos mentais constituem um grupo vulnerável e violações aos seus direitos humanos e liberdades básicas, bem como a negação dos seus direitos civis, políticos, sociais e culturais são uma ocorrência comum em todo o mundo, tanto dentro de instituições, como na comunidade ${ }^{(2)}$.

Dados da Organização Mundial da Saúde (OMS) indicam que os transtornos mentais são historicamente estigmatizados e os seus portadores estão entre as populações mais marginalizadas da maioria dos países ${ }^{(2)}$. Nesse contexto, observa-se que o exercício de direitos não é homogêneo para todas as pessoas, apesar de a Declaração Universal dos Direitos Humanos(3) e diferentes tratados internacionais produzidos com base em seu conteúdo, que é considerado um marco para a proteção dos direitos humanos, dispor em seu artigo $2^{\circ}$ que "toda pessoa tem capacidade para gozar os direitos e as liberdades estabelecidos nesta Declaração, sem distinção de qualquer espécie, seja de raça, cor, sexo, língua, religião, opinião política ou de outra natureza, origem nacional ou social, riqueza, nascimento, ou qualquer outra condição"(3).

Todavia, apesar do estigma existente com relação ao portador de transtorno mental, gradativamente se tem observado um movimento de mudança, considerando as especificidades do transtorno mental, reconhecendo os direitos humanos de seus portadores e enfatizando a necessidade de proteção do Estado(4). Esse movimento fortaleceu-se inicialmente na Itália e na década de 1980 emergiu no Brasil, sendo conhecido como Reforma Psiquiátrica.

A Reforma Psiquiátrica brasileira representa, portanto, um movimento político e social complexo, composto de atores, instituições e forças de diferentes origens $^{(5)}$. Do ponto de vista prático, a reforma da assistência proporcionou redução de internações psiquiátricas, desospitalização e criação de serviços comunitários que foram estruturados para atender aos portadores de transtornos mentais, os quais, até então, tinham como referência apenas o modelo assistencial hospitalocêntrico(6). Foi efetivada em 2001, com a Lei
Federal 10.216 que dispôs sobre a proteção e os direitos das pessoas portadoras de transtornos mentais, conferindo sustentação ao movimento no país.

Como resultado desse movimento são criados novos cenários de prestação de cuidados aos portadores de transtornos mentais, como os Centros de Atenção Psicossocial (CAPS), os Centros de Convivência e Cultura, as unidades/leitos psiquiátricos em hospitais gerais, o programa "De volta para casa", o Programa Nacional de Avaliação do Sistema Hospitalar/Psiquiatria e os Serviços Residenciais Terapêuticos. Dentre os equipamentos extra-hospitalares, este artigo destaca os Centros de Atenção Psicossocial (CAPS), regulamentados pela Portaria 336/2002.

No que diz respeito aos direitos dos portadores de transtornos mentais, a Lei 10.216 enfoca suas relações com os serviços de saúde, enfatizando nos artigos $1^{\circ}$ e $2^{\circ}$ : I - ter acesso ao melhor tratamento do sistema de saúde, consentâneo às suas necessidades; II - ser tratada com humanidade e respeito e no interesse exclusivo de beneficiar sua saúde, visando alcançar sua recuperação pela inserção na família, no trabalho e na comunidade; III - ser protegida contra qualquer forma de abuso e exploração; IV - ter garantia de sigilo nas informações prestadas; $V$ - ter direito à presença médica, em qualquer tempo, para esclarecer a necessidade ou não de sua hospitalização involuntária; VI - ter livre acesso aos meios de comunicação disponíveis; VII - receber o maior número de informações a respeito de sua doença e de seu tratamento; VIII - ser tratada em ambiente terapêutico pelos meios menos invasivos possíveis; IX - ser tratada, preferencialmente, em serviços comunitários de saúde mental(7). Ainda, durante os atendimentos em saúde mental, de qualquer natureza, a pessoa e seus familiares devem ser devidamente informados desses direitos. 0 Estado deve, também, se responsabilizar pela efetivação e pelo desenvolvimento de políticas que viabilizem o alcance da reinserção social do portador de transtorno mental( 7 .

Sendo assim, em busca de uma sociedade livre de preconceito, é necessário tratar as pessoas com transtornos mentais de forma igualitária e sem discriminação, com o objetivo de evitar seu sofrimento e garantir o direito ao tratamento adequado. Para tanto, os profissionais de saúde são atores fundamentais no processo de consolidação da reforma psiquiátrica no país, 
visando à garantia da igualdade de oportunidades e tratamento.

Nesse cenário, este estudo qualitativo objetivou descrever a percepção dos profissionais de saúde sobre os direitos humanos dos portadores de transtornos mentais e os meios para a efetivação desses direitos durante o cuidado à saúde.

\section{MÉTODOS}

Trata-se de pesquisa descritiva com abordagem qualitativa. Os dados foram coletados por meio de entrevistas semiestruturadas com profissionais da equipe de saúde de um CAPS do interior do estado de São Paulo. As entrevistas foram realizadas nos meses de fevereiro, março e abril de 2010 . Os 14 profissionais de saúde do serviço estudado foram convidados a participar da pesquisa, dentre os quais, sete aceitaram o convite e se tornaram sujeitos desta investigação.

O conteúdo das entrevistas abrangia o significado atribuído pelos profissionais de saúde aos direitos humanos dos portadores de transtornos mentais e meios para sua efetivação durante o cuidado à saúde.

As entrevistas foram gravadas, posteriormente transcritas e analisadas por meio da análise de conteúdo. A análise de conteúdo constitui um conjunto de técnicas de análise de comunicações, visando obter a inferência de conhecimentos relativos às condições de produção e recepção destas mensagens ${ }^{(8)}$.

A análise de dados foi realizada, em três fases: a préanálise, com a leitura e transcrição dos dados; a fase de exploração do material, com a organização dos dados em categorias temáticas. Рara isso, realizou-se a leitura exaustiva e repetida do conteúdo das entrevistas e dos recortes que representavam significado para responder os objetivos do estudo. Por fim, na terceira fase, foi realizado o tratamento e interpretação dos resultados analisados, com subsídio na literatura publicada sobre os direitos humanos dos portadores de transtornos mentais.

O projeto foi desenvolvido após sua aprovação por Comitê de Ética em Pesquisa (Protocolo n 1038/2009), de acordo com as regras da Resolução nº 196/96. A pesquisa foi conduzida de acordo com os padrões éticos exigidos, tendo sido solicitado aos participantes, depois de explicação detalhada dos objetivos do estudo, que assinassem o termo de consentimento esclarecido após sua aceitação explícita em participarem da pesquisa.

\section{RESULTADOS E DISCUSSÃO}

Participaram desta pesquisa sete profissionais de saúde, cinco mulheres e dois homens. Dentre eles, seis eram solteiros e um casado. Quanto à profissão, foram entrevistados dois enfermeiros, dois médicos psiquiatras, um auxiliar de enfermagem, um músico terapeuta e um assistente social.

Da análise de conteúdo resultaram as seguintes categorias temáticas: "direitos humanos dos portadores de transtornos mentais: foco na liberdade, proteção à vida, convivência em sociedade e exercício de cidadania", "meios para a concretização dos direitos humanos dos portadores de transtornos mentais no contexto do cuidado à saúde: informação, conscientização sobre direitos e incentivo à autonomia", "dificuldades vivenciadas para a concretização dos direitos humanos dos portadores de transtornos mentais no contexto do cuidado à saúde: número insuficiente de profissionais de saúde, espaço físico inadequado" e "ultrapassando as fronteiras do CAPS: sociedade, governo e família na concretização dos direitos humanos dos portadores de transtornos mentais".

Direitos humanos dos portadores de transtornos mentais: foco na liberdade, proteção à vida, convivência em sociedade e exercício de cidadania

As entrevistas revelaram diferentes percepções dos profissionais de saúde sobre os direitos humanos dos portadores de transtornos mentais, dentre elas, os sujeitos associaram os direitos à liberdade e à proteção da vida destas pessoas.

[...] Que protege nossa vida, direito à liberdade, de andar. (E2)

\section{[...] É o direito à vida. (E7)}

Define-se liberdade como ausência de interferência na vida do indivíduo, permitindo o exercício da autonomia $^{(9)}$. De forma geral, a convivência em sociedade depende da defesa da liberdade e integridade da vida, considerados direitos humanos fundamentais. Nessa perspectiva, no caso dos portadores de transtornos mentais, podem ser estabelecidos limites a sua liberdade e autonomia, em situações em que o transtorno impede o sujeito de praticar os atos da vida civil e responder pelas consequências advindas do seu agir ${ }^{(9)}$. 
Em contraposição, se a pessoa não está incapacitada para exercer suas vontades e sua autonomia, pode aceitar ou mesmo negar qualquer intervenção médica, garantindo sua liberdade e dignidade. Baseado nos princípios da autonomia e dignidade da pessoa humana, os valores principais em qualquer tratamento médico consistem no bem estar do paciente e no respeito a sua pessoa ${ }^{(10)}$.

Todos os profissionais que prestam cuidado a um paciente com transtorno mental deverão tratá-lo acima de tudo com humanidade e respeito. Na relação médicopaciente, o respeito do primeiro à autonomia do segundo representa o respeito à dignidade humana em toda a sua essência. A autonomia do indivíduo pressupõe, portanto, a capacidade que têm as pessoas para a sua autodeterminação no que concerne às opções individuais de que dispõe, o respeito a sua vontade, autogoverno e participação no processo terapêutico(11).

Ressalte-se, ainda, que, considerando que o estigma relacionado ao transtorno mental resultou historicamente em uma série de infrações aos direitos humanos de seus portadores, especialmente do seu direito à liberdade e proteção da vida, é ainda mais relevante o papel dos profissionais de saúde durante o cuidado, buscando valorizar sua liberdade, sem colocar em risco sua integridade física.

Nesse sentido, os profissionais de saúde relacionaram a importância dos direitos dos portadores de transtornos mentais, como base para sua convivência em sociedade, assim como garantia ao exercício de sua cidadania.

[...] engloba maneiras da pessoa viver dentro de uma sociedade. [...] (E4)

\section{[...] Direito a ser tratado como cidadão. (E5)}

[...] Um direito humano é (...) garantia de (...) cidadania [...] (E6)

No contexto do CAPS, a ênfase na convivência social, reafirma os direitos humanos de cidadania dos portadores de transtornos mentais. O cidadão possui o legado de resolver problemas sociais e questões públicas, deliberando e debatendo sobre elas, buscando os seus direitos e interagindo com os demais cidadãos ${ }^{(12)}$. Dessa forma, efetivar a possibilidade de exercício de sua cidadania pelos portadores de transtornos mentais depende da geração de um poder cívico, que está diretamente relacionado à construção da capacidade de convivência em sociedade.

Vista como um processo histórico de conquistas populares, a cidadania é o meio pelo qual a sociedade se torna organizada, com capacidade para desenvolver e concretizar um projeto próprio de desenvolvimento. 0 resgate da cidadania dos portadores de transtornos mentais deve ser uma meta dos profissionais de saúde, usuários e sociedade, concretizada por meio da reabilitação psicossocial. Pode-se afirmar que se insere no âmbito do direito de cidadania do portador de transtorno mental o direito de receber assistência adequada e a garantia de participar da sociedade ${ }^{(13)}$.

Nesse cenário, estudos mostram que os CAPS têm atuado efetivamente no tratamento, aliando acompanhamento clínico e cuidados de reinserção social dos usuários por meio do acesso ao trabalho e ao lazer, bem como ao exercício dos direitos civis e à construção e reconstrução de laços familiares e comunitários ${ }^{(14)}$.

\section{Meios para a concretização dos direitos humanos dos portadores de transtornos mentais no contexto do cuidado à saúde: informação, conscientização sobre direitos e incentivo à autonomia}

Informar e conscientizar o usuário do CAPS e familiares sobre seus direitos, assim como a população em geral, demonstrando que são direitos necessários e importantes a todos, foram meios discutidos pelos entrevistados para a concretização dos direitos humanos dos portadores de transtornos mentais.

[...] Acho que inclusive a maior concretização seria a conscientização das pessoas que elas têm esses direitos, elas não sabem disso né?[...] (E5)

[...] A gente sempre tenta respeitar esse direito à saúde, trabalhando mais com o paciente, envolvendo a família dele, para ele poder se conscientizar do que é um direito [...] (E1)

Para que a participação do cidadão na sociedade seja efetivada, a informação se torna um elemento de fundamental importância no âmbito do exercício dos direitos humanos, pois, desse modo, os sujeitos tomam conhecimento de seus direitos e deveres e começam a tomar decisões sobre suas vidas, de forma individual ou 
coletiva $^{(15)}$. A cidadania e o processo de sua construção perpassam o acesso à informação, pois as conquistas de direitos políticos, civis e sociais e a responsabilização quanto aos deveres dependem da sua disseminação e circulação, resultando em reflexões críticas quanto às questões sobre a construção de uma sociedade mais justa e com maiores oportunidades para os cidadãos.

Nessa perspectiva, salienta-se o CAPS, como instituição disseminadora de informações sobre os direitos dos usuários do serviço, enfatizando-se a importância dos profissionais da equipe na conscientização dos usuários e seus familiares sobre os seus direitos e possibilidades de exercício de sua cidadania. A informação é, então, a base para a conscientização e meio para o empoderamento dos portadores de transtornos mentais.

Os sujeitos descreveram, também, que o CAPS trabalha envolvendo o paciente e a família com o objetivo de reinserção social, valorizando a autonomia e independência do portador de transtorno mental, visando à efetivação dos direitos humanos desses usuários.

[...] é lá fora, é na sociedade, e a gente tenta trabalhar com a reinserção social, a gente procura buscar outros meios de (...) é, não dependendo só do CAPS, só das atividades daqui, mas tentando envolver em outras atividades lá fora, para buscar mais autonomia e independência [...] (E3)

A reinserção social representa um dos pilares da Reforma Psiquiátrica, sendo considerada uma estratégia global que implica o envolvimento dos portadores de transtornos mentais, seus familiares, profissionais de saúde mental, gestores e a comunidade. Nesse processo, a manutenção ou restabelecimento dos vínculos familiares e sociais representa um grande desafio. A Lei $10.216^{(7)}$ assegura às pessoas com transtornos mentais o direito ao convívio familiar, em conformidade com o disposto na Constituição Federal, no seu art. 226. Esse dispositivo embasa-se não somente na observância de um direito fundamental, mas, sobretudo, no entendimento dos profissionais de saúde de que as relações familiares podem contribuir para a saúde mental dos portadores de transtornos mentais.

Como pressuposto para a reinserção social, a vida em comunidade corresponde a um direito assegurado pela
Lei 10.216, em consonância com o Princípio 3 dos “Princípios para a Proteção de Pessoas Acometidas de Transtorno Mental e para a Melhoria da Assistência à Saúde Mental"(16). Outrossim, apesar da positivação dos direitos humanos dos portadores de transtornos mentais e a sua concretização na política de saúde mental do país, são ainda necessárias muitas conquistas e mudanças para que estes direitos realmente sejam consolidados em suas vidas.

\section{Dificuldades vivenciadas para a concretização dos direitos humanos dos portadores de transtornos mentais no contexto do cuidado à saúde: número insuficiente de profissionais de saúde, espaço físico inadequado}

Ao descreverem os meios para a concretização dos direitos humanos, os sujeitos relataram algumas dificuldades, como o número insuficiente de profissionais e o espaço físico inadequado, como dificultadores do bom atendimento e entraves para uma atuação mais efetiva dos profissionais de saúde.

[...] Acho que deveria aumentar o número de profissionais, acho que são poucos profissionais, né? Acho que poderia aumentar porque é melhor para os pacientes... às vezes falta espaço físico também[...](E2)

[...] um espaço melhor, que a equipe fosse mais completa, pois é muito reduzida [...] (E7)

As falas demonstram que as instituições de saúde ainda não oferecem um ambiente adequado, recursos humanos e materiais suficientes, o que dificulta aos profissionais atuarem mais motivados ${ }^{(17-18)}$. A parte física constitui importante fator para o preparo de um ambiente humano, que interfere na qualidade do atendimento, e, muitas vezes, impede ou impossibilita alguns procedimentos ${ }^{(18)}$.

Sendo assim, é necessário tratar a adequação da estrutura física e de recursos humanos do CAPS como prioridade, buscando-se uma resolução política no contexto da saúde mental e pelos órgãos responsáveis no município e outras instâncias de decisão(19). 
Ultrapassando as fronteiras do CAPS: sociedade, governo e família na concretização dos direitos humanos dos portadores de transtornos mentais

Os participantes do estudo reafirmaram a relevância do envolvimento do governo e da família para a consolidação dos direitos dos portadores de transtornos mentais.

[...] Que ainda falta muito com relação aos direitos, principalmente em relação ao portador de deficiência mental no caso, acho que deixa a desejar demais, tanto pela sociedade, pelo governo [...] (E1)

A luta pelos direitos humanos resulta de um processo histórico e dinâmico de conquistas e de consolidação de espaços emancipatórios da dignidade humana ${ }^{(20)}$, no qual - Estado e a sociedade civil têm responsabilidades necessariamente compartilhadas. A Reforma Psiquiátrica brasileira representa significativo exemplo de luta fomentada pelos trabalhadores de saúde mental e de segmentos da sociedade civil pelos direitos humanos dos portadores de transtornos mentais.

Ao citarem possibilidades de concretização desses direitos, os entrevistados ressaltaram o papel da sociedade, governo e família. Enfatiza-se, então, a coresponsabilidade da família no tratamento do portador de transtorno mental.

A vivência com o portador de transtorno mental, muitas vezes é considerada tarefa difícil, devido ao seu comportamento em alguns casos imprevisível, agressivo ou isolado, levando ao desgaste dos familiares e receio da sociedade. Portanto, os profissionais de saúde devem, sempre que possível, envolver a família e encorajar o portador de transtorno mental a compreender que a família deve participar em ações de formação(21).

Todavia, convocar a família para assumir a responsabilidade do cuidado do usuário em conjunto com a equipe exige mais do que um redirecionamento das práticas. Requer comprometimento com a construção de um cuidado coletivo provedor da autonomia do usuário, auxiliando-o a reconquistar seu espaço na sociedade.

Apesar das possibilidades, os participantes percebem dificuldades para que ocorra o envolvimento da família e sociedade, em virtude do preconceito com relação ao transtorno mental e o seu portador.
[...] Ainda falta muito esclarecimento da população, da família que atende. As famílias não têm respaldo em casa, muitos não conseguem entender como é a doença e o tratamento. Às vezes não é nem culpada a família é porque eles não têm noção de como podem ajudar [...] (E4).

As famílias que convivem com uma situação crônica de doença continuam com as mesmas funções desempenhadas por outras famílias, porém lhes é acrescida mais uma atribuição, o cuidar na doença. Tais famílias estão em situação de vulnerabilidade, pois a doença crônica, dada suas características e quando não devidamente controlada, suga as energias da família e transforma seu contexto e cotidiano(22).

Os profissionais de saúde precisam atentar-se para as dificuldades enfrentadas por algumas famílias em lidar com paciente diagnosticado com transtorno mental. Os preconceitos que sofre o usuário vão se refletir na família, que pode, por isso, negar a doença ou abandonar o paciente. A efetiva participação da família como grupo mais importante na vida do portador de transtorno mental é uma ferramenta fundamental para o sucesso do tratamento. Em suma, somente com o envolvimento do profissional e da família haverá possibilidade de o processo de desinstitucionalização e reinserção do portador de transtorno mental ocorrer de forma satisfatória(23), favorecendo o exercício de sua cidadania.

\section{CONCLUSÕES}

O movimento de evolução dos direitos humanos demonstra um processo de construção constante, marcado por crescentes lutas da população. Apesar da positivação dos direitos humanos no Brasil, constatam-se várias lacunas em sua aplicação e consolidação, especialmente por grupos vulneráveis como os portadores de transtornos mentais. Considerando a relevância da garantia dos direitos humanos dos portadores de transtornos mentais, este estudo qualitativo buscou descrever a percepção dos profissionais de saúde de um CAPS do interior paulista sobre os direitos humanos destes pacientes e meios para sua consolidação no contexto do cuidado à saúde.

Os resultados dessa investigação demonstram uma compreensão do direito como meio para se buscar maior simetria nas relações sociais. A Lei 10.216 representa um marco no redirecionamento dos cuidados à saúde dos 
portadores de transtornos mentais no país, enfatizando seus direitos durante a assistência à saúde mental como condição de resgate de sua cidadania. Dessa forma, a percepção dos participantes do estudo associa o direito ao ideal de liberdade e à possibilidade de convivência social. Nesse sentido, os direitos humanos somente são incorporados ao patrimônio individual por meio do pertencimento a uma comunidade, o que muitas vezes é dificultado pela exclusão social a que estão sujeitos os portadores de transtornos mentais.

Este contexto realça ainda mais o importante papel que pode ser desempenhado pelos profissionais de saúde e pelo CAPS, especialmente na disseminação de

\section{REFERÊNCIAS}

1. Ventura CAA, Mello DF, Andrade RD, Mendes IAC. Aliança da enfermagem com o usuário na defesa do SUS. Rev Bras Enferm [Internet]. 2012 [acesso em: 20 dez 2013];65(6):893-8.

Disponivel em: http://dx.doi.org/10.1590/S003471672012000600002 .

2. Organização Mundial da Saúde. Livro de Recursos Humanos da OMS sobre Saúde Mental, Direitos Humanos e Legislação. Cuidar sim, Excluir não [Internet]. Genebra: Organização Mundial de Saúde; 2005 [acesso em: 20 dez 2013]. Disponível em:

http://www.who.int/mental health/policy/Livroderecursosrevis ao FINAL.pdf.

3. Assembléia Geral das Nações Unidas, Organização das Nações Unidas. Declaração Universal dos Direitos Humanos. Nova Iorque: Organização das Nações Unidas; 1948.

4. Hunt $P$, Mequita J. Mental Disabilities and the Human Right to the Highest Attainable Standard of Health. Hum Rights Q [Internet]. 2006 [acesso em: $20 \mathrm{dez}$ 2013];28(2):332-56. Disponivel em: http://dx.doi.org/10.1353/hrq.2006.0019. 5. Ministério da Saúde. Reforma psiquiátrica e política de saúde mental no Brasil. Documento apresentado à Conferência Regional de Reforma dos Serviços de Saúde Mental : 15 anos depois de Caracas [Internet]. Brasília: Ministério da Saúde, 2005 [acesso em: $20 \mathrm{dez}$ 2013]. Disponível em:

http://bvsms.saude.gov.br/bvs/publicacoes/relatorio15 anos c aracas.pdf.

6. Tansella M, Amaddeo F, Burti L, Lasalvia A, Ruggeri M.

Evaluating a community-based mental health service focusing on severe mental illness. The Verona experience. Acta Psychiatr Scand Suppl[Internet]. 2006 [acesso em: 20 dez 2013]; 113(Supl. 429):90-4. Disponível em: http://dx.doi.org/10.1111/j.16000447.2005.00724.x.

7. Lei $n^{\circ} 10.216$, de 6 de abril de 2001. Dispõe sobre a proteção e os direitos das pessoas portadoras de transtornos mentais e redireciona o modelo assistencial em saúde mental. Diário Oficial da União [Internet]. 09 abr. 2001 [acesso em: $20 \mathrm{dez}$ 2013]. Disponivel em:

http://www.planalto.gov.br/ccivil 03/leis/leis 2001/l10216.htm. 8. Bardin L. Análise de conteúdo. Rio de Janeiro: Edições 70; 1977.

9. Lopes JRL. Direitos humanos e tratamento igualitário: questões de impunidade, dignidade e liberdade. Rev. bras. Ci. Soc. [Internet]. 2000 [acesso em: 20 dez 2013];15(42):77-100. Disponível em: http://dx.doi.org/10.1590/S0102$\underline{69092000000100006}$. informação sobre direitos e no envolvimento da família, sempre com foco no respeito à dignidade humana dos seus usuários. A estrutura física do serviço e a limitação em termos de recursos humanos, em conjunto com o estigma vivenciado pelo paciente e seus familiares, representam graves entraves nesse processo de concretização de direitos.

Como perspectiva futura, é fundamental que se invista no empoderamento dos portadores de transtornos mentais, para que superem a posição de pacientes e possam agir como cidadãos, e, em conjunto com a equipe de saúde, trabalhem suas possibilidades de reinserção social e de exercício de sua autonomia.

10. Lara MC, Fuente JR. Sobre el consentimiento informado. Boletín de la Oficina Sanitaria Panamericana (OSP) [Internet]. 1990 [acesso em: 20 dez 2013];108(5-6):439-44. Disponível em: http://hist.library.paho.org/Spanish/BOL/v108n(5-6)p439.pdf. 11. Fortes HM. Tratamento compulsório e internações psiquiátricas. Rev. Bras. Saude Mater. Infant. [Internet]. 2010 [acesso em: 20 dez 2013];10 (Supl. 2):s321-30. Disponível em: http://dx.doi.org/10.1590/S1519-38292010000600009.

12. Simeone M. A cidadania como possibilidade. Revista Diversa [Internet]. 2005 [acesso em: $20 \mathrm{dez}$ 2013];3(8). Disponível em: http://www.ufmg.br/diversa/8/artigo-

acidadaniacomopossibilidade.htm.

13. Jorge MSB, Randemark NFR, Queiroz MVO, Ruiz EM.

Reabilitação Psicossocial: visão da equipe de Saúde Mental. Rev Bras Enferm [Internet]. 2006 [acesso em: $20 \mathrm{dez}$ 2013];59(6):734-9. Disponível em:

http://dx.doi.org/10.1590/50034-71672006000600003. 14. Cordeiro LRO, Oliveira MS, Souza RC. Produção científica sobre os Centros de Atenção Psicossocial. Rev Esc Enferm USP [Internet]. 2012 [acesso em: $20 \mathrm{dez} 2013$ ];46(1):119-23. Disponivel em: http://dx.doi.org/10.1590/S008062342012000100016.

15. Araujo EA. Informação, sociedade e cidadania: práticas informacionais de Organizações Não-Governamentais (ONGs) brasileiras. Informação \& Informação [Internet]. 2001 [acesso em: 20 dez 2013];6(1). Disponível em:

http://www.uel.br/revistas/uel/index.php/informacao/article/vi ew/1683/0.

16. Organização das Nações Unidas. Princípios para a Proteção de Pessoas Acometidas de Transtorno Mental e a Melhoria da Assistência à Saúde Mental. Nova lorque: Organização das Nações Unidas; 1991.

17. Macedo J, Silveira M, Eulálio M, Fraga M, Braga V. Representação Social da assistência de enfermagem em saúde mental: estudo qualitativo. Online braz j nurs [Internet]. 2010 [acesso em: $20 \mathrm{dez} 2013$ ];9(3) . Disponível em: http://dx.doi.org/10.5935/1676-4285.2007581.

18. Simões ALA, Rodrigues FR, Tavares DMS, Rodrigues LR. Humanização na saúde: enfoque na atenção primária. Texto Contexto Enferm [Internet]. 2007 [acesso em: $20 \mathrm{dez}$ 2013];16(3): 439-44. Disponível em:

http://dx.doi.org/10.1590/S0104-07072007000300009.

19. Olschowsky A, Glanzner CH, Mielke FB, Kantorski LP, Wetze C. Avaliação de um Centro de Atenção Psicossocial: a realidade em Foz do Iguaçu. Rev Esc Enferm USP [Internet]. 2009 [acesso 
em: 20 dez 2013];43(4):781-7. Disponível em:

http://dx.doi.org/10.1590/S0080-62342009000400007.

20. Baptista MV. Algumas reflexões sobre o sistema de garantia de direitos. Serv. Soc. Soc. [Internet]. 2012 [acesso em: 20 dez 2013];(109):179-99. Disponível em:

http://dx.doi.org/10.1590/S0101-66282012000100010.

21. Lefley HP. O papel das famílias na integração comunitária: a promoção de serviços de saúde mental, investigação e recovery. In.: Ornelas J, Monteiro FJ, Moniz MJV, Duarte T. Participação e Empowerment das Pessoas com Doença Mental e seus

Familiares [Internet]. Lisboa: AEIPS Edições; 2005 [acesso em: 20 dez 2013]. p. 57-80. Disponível em:

http://aeips.pt/novo2009/wp-

content/uploads/2009/07/part_empower.pdf.

22. Marcon SS, Radovanovic CAT, Waidman MAP, Oliveira MLF, Sales CA. Vivência e reflexões de um grupo de estudos junto às famílias que enfrentam a situação crônica de saúde. Texto Contexto Enferm [Internet]. 2005 [acesso em: $20 \mathrm{dez}$ 2013];14(n. esp.):116-24. Disponível em: http://dx.doi.org/10.1590/S0104-07072005000500015.

23. Silva KVL, Monteiro ARM. A família em saúde mental: subsídios para o cuidado clínico de enfermagem. Rev Esc Enferm USP [Internet]. 2011 [acesso em: $20 \mathrm{dez}$ 2013];45(5):1237-42. Disponível em: http://www.scielo.br/scielo.php?script=sci_arttext\&pid=S008062342011000500029\&lng=en\&nrm=iso.

Artigo recebido em 21/08/2012.

Aprovado para publicação em 12/09/2013.

Artigo publicado em 31/12/2013. 